\title{
The interactions of cobalt(II) with mitochondria from rat liver
}

\author{
Marcantonio Bragadin · Antonio Toninello • \\ Mario Mancon · Sabrina Manente
}

Received: 10 October 2006/ Accepted: 22 January 2007

(C) SBIC 2007

\begin{abstract}
The interactions of $\mathrm{Co}^{2+}$ with mitochondria have been investigated. The results indicate that $\mathrm{Co}^{2+}$ inhibits ATP synthesis. Further investigations into ATP synthesis mechanisms indicated that inhibition is due to the opening of a transmembrane pore. The opening of this pore causes the collapse of the high-energy intermediate where, under a $\mathrm{pH}$ and a potential gradient, the energy is stored and subsequently utilized to form ATP from ADP.
\end{abstract}

Keywords Cobalt $\cdot$ Mitochondria $\cdot$ Energy synthesis

\section{Introduction}

Cobalt has both beneficial and harmful effects on human health. It is beneficial for humans because it is a part of vitamin $\mathrm{B}_{12}$, which is essential for the maintenance of human health. When too much cobalt is taken into the body, it can produce harmful effects, which have been the subject of many in vivo studies. In vitro investigations have also been performed, in order to establish the molecular mechanism responsible for these effects on whole organisms. However, the exact molecular mechanism that $\mathrm{Co}^{2+}$ exerts on cells has still not been identified, although a number of potential mechanisms have been proposed [1$3]$. The aim of the work presented here was not to carry out

M. Bragadin $(\varangle) \cdot$ S. Manente

Dipartimento di Scienze Ambientali,

Università Cà Foscari,

Venezia DD 2137, 30123 Venice, Italy

e-mail: bragadin@unive.it

A. Toninello $\cdot$ M. Mancon

Dipartimento di Chimica Biologica,

Università di Padova e Centro Studio delle Biomembrane,

C.N.R. Padova, Viale G. Colombo 3, 35121 Padua, Italy a toxicological investigation, but rather a study of the molecular mechanisms of the interaction of $\mathrm{Co}^{2+}$ with biological structures, which can subsequently be utilized for toxicological studies.

Since, in many cases, $\mathrm{Co}^{2+}$ causes apoptosis in isolated cells $[4,5]$, and since the apoptosis can be correlated with mitochondrial function, we investigated the interactions of $\mathrm{Co}^{2+}$ with mitochondria. The results indicated that $\mathrm{Co}^{2+}$ actually does inhibit ATP synthesis, and further investigations were then carried out to identify the step responsible for ATP synthesis inhibition. This step appears to be the opening of a membrane pore which reduces the energy accumulation in the mitochondria.

\section{Materials and methods}

The mitochondria were prepared from the livers of fasted albino Wistar rats, weighing about $300 \mathrm{~g}$ [7]. Mitochondrial protein was determined using the Lowry procedure [8].

The mitochondrial oxygen consumption was measured using a Clark oxygen electrode (Yellow Springs Instruments, Yellow Springs, OH, USA) fitted in a thermostatcontrolled, closed chamber with a magnetic stirrer. The reaction medium $(2 \mathrm{ml})$ was maintained at $25{ }^{\circ} \mathrm{C}$ throughout all the experiments (i.e., under standard in vitro conditions).

The following reducing substrates were utilized: succinate, glutamate/malate and ascorbate $/ N, N, N^{\prime}, N^{\prime}$-tetramethyl-p-phenylenediamine (TMPD).

To follow the absorbance decrease, the swelling experiments were performed at $540 \mathrm{~nm}$, using a Jenway 6400 (Felsted, UK) spectrophotometer, equipped with stirring apparatus. After the addition of the mitochondria to the resuspending medium $(2.5 \mathrm{ml})$, the instrument was adjusted to zero absorbance. 
The ATP synthesis/hydrolysis experiments were performed in a low-buffered medium, using a $\mathrm{pH}$ electrode connected to an 84 PHM Radiometer ( $\mathrm{pH}$ meter) (Radiometer, Copenhagen, Denmark) in order to monitor the $\mathrm{pH}$ changes which accompany the reaction

$\mathrm{ADP}+\mathrm{Pi}+\mathrm{H}^{+} \leftrightarrow \mathrm{ATP}$

where Pi is sodium phosphate, under stirring conditions, at room temperature.

All of the reagents were of analytical grade. 2,4-Dinitrophenol (DNP), sodium succinate, sodium glutamate, sodium malate, oligomycin, and TMPD were obtained from Sigma (Milan, Italy).

\section{Results}

In mitochondria, the substrates arising from the Krebs cycle are oxidized by molecular oxygen, and ATP is produced. This oxidation occurs by means of a sequence of redox couples, called the mitochondrial respiratory chain (RC). As envisaged in the chemiosmotic hypothesis [9], the electron flow in the RC is coupled to a proton extrusion. Since the (inner) mitochondrial membrane is not permeable to protons, their extrusion gives rise to a $\Delta \mathrm{pH}$ and a $\Delta \Psi$. The sum of these two components is called the proton motive force (pmf):

$\mathrm{pmf}=\Delta \mathrm{pH}+\Delta \Psi$

Under this form, the free energy arising from the oxidation of the substrates is stored and subsequently utilized to synthesize ATP from ADP.

As a consequence of the mechanism discussed above, inhibition of the ATP synthesis can be due to:

- The inhibition of ATPase (the enzyme that catalyzes the ATP synthesis)

- Inhibition of the RC

- Enhancement of the membrane proton permeability

Therefore, as it has been proven that $\mathrm{Co}^{2+}$ inhibits ATP synthesis (Fig. 1) (although this inhibition, in the presence of phosphate, could be due to the formation of the $\mathrm{CoHPO}_{4}$ complex [10]), each step involved in ATP synthesis was analyzed separately, in order to identify the step that requires the lowest dose of $\mathrm{Co}^{2+}$ needed to inhibit the ATP synthesis.

\section{ATPase}

As ATPase is the enzyme which catalyzes ATP synthesis, its inhibition implies a corresponding inhibition of ATP synthesis.
As indicated in Fig. 1, the inhibition of ATP synthesis by $\mathrm{Co}^{2+}$ is not accompanied by an inhibition of ATP hydrolysis following the production of ATP. This type of behavior was further confirmed by means of direct experiments concerning ATP hydrolysis which can be seen in Fig. 2. The addition of ATP to the uncoupled mitochondria gives rise to a rapid acidification in the (low-buffered) medium. This acidification is a consequence of the following reaction:

$\mathrm{ATP} \rightarrow \mathrm{ADP}+\mathrm{Pi}+\mathrm{H}^{+}$

The addition of oligomycin (Fig. 2, reaction b), which is an ATPase inhibitor, stops the process of ATP hydrolysis, while the addition of $\mathrm{Co}^{2+}$, up to a concentration of $300 \mu \mathrm{M}$, does not induce a cessation in the acidification (Fig. 2, reaction a). This leads to the conclusion that $\mathrm{Co}^{2+}$ is not an ATPase inhibitor.

\section{The RC}

As the $\mathrm{RC}$ is correlated to the proton extrusion, an inhibition of the RC implies an equivalent inhibition of ATP synthesis. The interactions of $\mathrm{Co}^{2+}$ with the $\mathrm{RC}$ were investigated by stimulating respiring mitochondria by adding ADP and Pi (this was possible because the previous experiments had demonstrated that $\mathrm{Co}^{2+}$ is not an ATPase

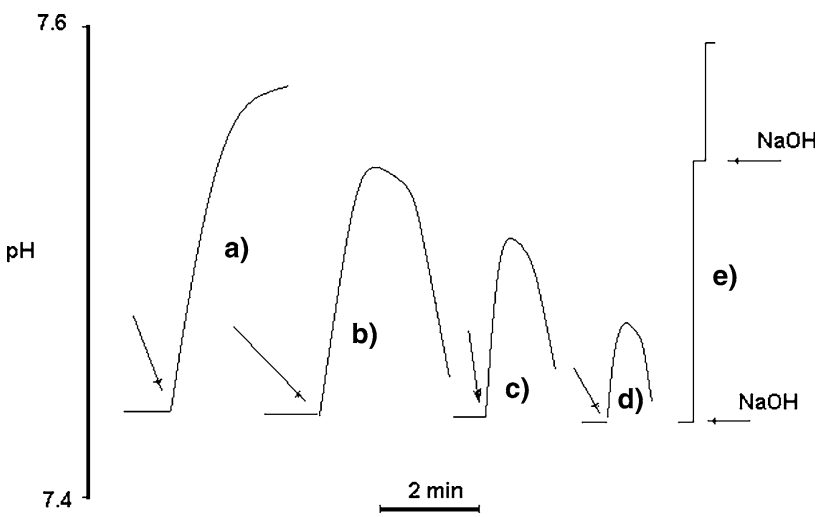

Fig. $1 \mathrm{Co}^{2+}$ inhibits ATP synthesis in mitochondria. The medium composition was $0.25 \mathrm{M}$ sucrose, $0.1 \mathrm{mM} N$-(2-hydroxyethyl)piperazine- $N$-ethanesulfonic acid (Hepes)/3-( $N$-morpholino)propanesulfonic acid (Mops) $\mathrm{pH} 7.4,1 \mathrm{mM}$ succinate, $1 \mathrm{mM} \mathrm{MgCl}_{2}, 0.5 \mathrm{mM}$ sodium phosphate $(\mathrm{Pi})$, and $0.5 \mathrm{mM}$ ADP. The final concentration of mitochondria was $0.5 \mathrm{mg} / \mathrm{ml}$. Since the ATP synthesis induces alkalinization, the reaction was followed using a $\mathrm{pH}$ meter in a low-buffered medium. The addition of $0 \mu \mathrm{M}(a), 5 \mu \mathrm{M}(b), 10 \mu \mathrm{M}$ $(c)$, and $15 \mu \mathrm{M}(d) \mathrm{Co}^{2+}$ induces a subsequent inhibition of the alkalinization, which demonstrates that $\mathrm{Co}^{2+}$ inhibits the ATP synthesis. The arrows indicate the addition of succinate (or $\mathrm{NaOH}$ in $e$ ). In $e$, each arrow indicates the addition of $5 \mathrm{nmol} \mathrm{NaOH}$ to the medium containing the mitochondria 

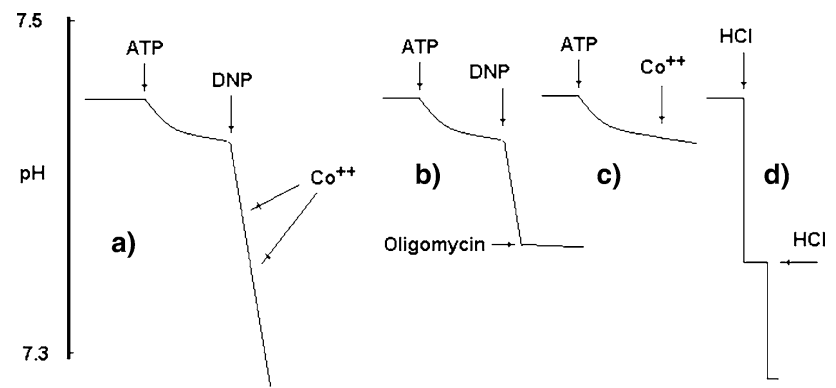

$2 \min$

Fig. $2 \mathrm{Co}^{2+}$ does not inhibit ATP hydrolysis in mitochondria. The medium composition was $0.25 \mathrm{M}$ sucrose, $0.1 \mathrm{mM}$ Hepes/Mops $\mathrm{pH}$ $7.4,1 \mu \mathrm{g} / \mathrm{ml}$ cyclosporine, $1 \mathrm{mM} \mathrm{MgCl} 2,1 \mathrm{mM}$ ATP, and $0.1 \mathrm{mM} \mathrm{Pi}$. The final concentration of mitochondria was $0.5 \mathrm{mg} / \mathrm{ml}$. ATP hydrolysis is facilitated by the addition of an uncoupler $(0.1 \mathrm{mM}$ 2,4-dinitrophenol). Under these conditions, $a$ the rapid ATP hydrolysis is not inhibited by successive additions of $\mathrm{Co}^{2+}$ up to $0.3 \mathrm{mM}$. The effects of the addition of oligomycin $(1 \mu \mathrm{g} / \mathrm{ml})$, a potent ATPase inhibitor, are shown for comparison purposes in $b$. In $c$ the addition of $0.2 \mathrm{mM} \mathrm{Co}^{2+}$ clearly shows that $\mathrm{Co}^{2+}$ is not an uncoupler. In $d$, each arrow indicates the addition of $2 \mathrm{nmol} \mathrm{HCl}$ to the medium containing the mitochondria

inhibitor). Under these conditions (Fig. 3 shows a typical experiment), the addition of $\mathrm{Co}^{2+}$ induces an inhibition of the respiratory rate when succinate is used as a reducing substrate. The graph in Fig. 3 shows the quantitative inhibition of the RC by $\mathrm{Co}^{2+}$, but it is evident from the graph that the doses necessary to induce inhibition of the RC are much higher than those needed to inhibit ATP synthesis. When the reducing substrate is either glutamate/ malate or ascorbate/TMPD, no RC inhibition occurs (not shown). Therefore, the possibility that the inhibition of the $\mathrm{RC}$ by $\mathrm{Co}^{2+}$ produces inhibition of ATP synthesis can be excluded.

The enhancement of membrane permeability

The crucial point of the chemiosmotic hypothesis is that the mitochondrial membrane is not permeable to protons; therefore, any mechanism which enhances proton permeability gives rise to an equivalent inhibition of ATP synthesis.

An enhancement of the membrane permeability to protons is called an uncoupling, or a protonophore, effect. A protonophore must be a weak acid (or base). Take, for example, DNP, which was the first protonophore studied [9]. It enters into the matrix as an undissociated compound, the driving force being the internal alkaline $\mathrm{pH}$. Once inside, DNP is extruded as a phenate anion by means of the negative internal potential. The resulting cyclic process implies the entry of a proton at each cycle.

Such a mechanism is, in theory, possible with $\mathrm{Co}^{2+}$, as it is a weak acid, but the experiments reported in Fig. 2,
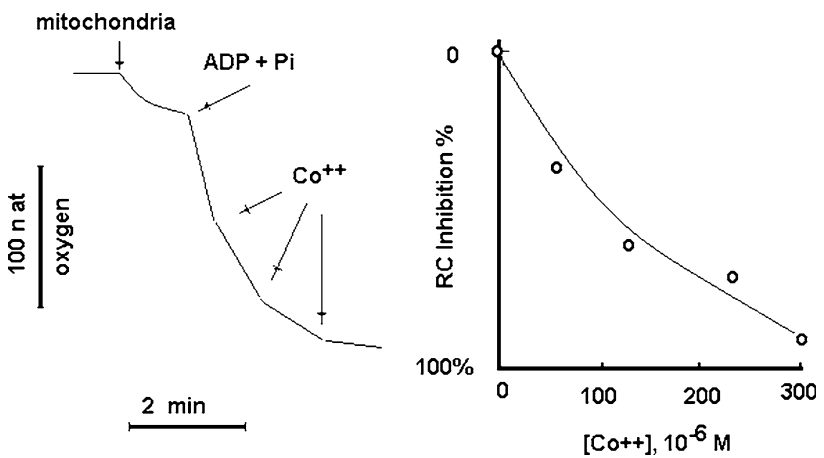

Fig. $3 \mathrm{Co}^{2+}$ inhibits the respiratory chain $(R C)$. The medium composition was $0.25 \mathrm{M}$ sucrose, $10 \mathrm{mM}$ Hepes/Mops $\mathrm{pH}$ 7.4, $1 \mathrm{mM} \mathrm{MgCl} 2$, and $1 \mathrm{mM}$ succinate. The final concentration of mitochondria was $0.5 \mathrm{mg} / \mathrm{ml}$. The addition of $0.5 \mathrm{mM}$ Pi and ADP induces a respiratory rate increase. Under these conditions, successive additions of $\mathrm{Co}^{2+}$ induce an inhibition (typical experiment). The graph shows the percentage of respiratory rate inhibition as a function of the amounts of $\mathrm{Co}^{2+}$ added

reaction c exclude this possibility, as it was observed that the mitochondria were energized by ATP. Under these conditions, a steady state occurs between the ATP and the pmf,

$\mathrm{ATP} \leftrightarrow \Delta \mathrm{pH}+\Delta \Psi$,

and, for this reason, in the absence of an uncoupler, the rate of ATP hydrolysis is very slow and, consequently, the acidification rate is also very slow.

In the presence of an uncoupler, both $\Delta \mathrm{pH}$ and $\Delta \Psi$ collapse, and a rapid ATP hydrolysis takes place. This hydrolysis can be easily monitored by a $\mathrm{pH}$ change in a low-buffered medium (in the same way as in the experiments reported in Fig. 1). In contrast to DNP, $\mathrm{Co}^{2+}$ does not stimulate ATP hydrolysis (Fig. 2, reaction c). This leads to the conclusion that $\mathrm{Co}^{2+}$ is neither an uncoupler nor, for similar reasons, a detergent, since a detergent compound renders permeable the membrane to all solutes, including protons.

Another mechanism which enhances the membrane permeability is the opening of a membrane pore (MTP pore).

Many chemical and toxic compounds induce the opening of a membrane pore. The size of this pore allows the passage of large molecules $[10,11]$. If the mitochondria are resuspended in a sucrose medium, the opening of the pore allows the entry of sucrose. The latter occurs in conjunction with the entry of water and, as a consequence of a colloid-osmotic effect, membrane swelling occurs [10]. This swelling, which is inhibited by cyclosporine [10, 11], can be identified by means of absorbance quenching at $540 \mathrm{~nm}$. Figure 4 shows both the swelling induced by $\mathrm{Co}^{2+}$ and its inhibition by cyclosporine. These examples demonstrate that $\mathrm{Co}^{2+}$ induces the opening of the MTP pore. In 
addition, the doses necessary to induce this phenomenon are very similar to those needed to inhibit ATP synthesis (Fig. 1).

Therefore, we have concluded that the inhibition of ATP synthesis by $\mathrm{Co}^{2+}$ is a consequence of the opening of the MTP pore, which reduces the energy in the mitochondria, stored in the form of a proton gradient $(\Delta \mathrm{pH}+\Delta \Psi)$. It should be noted, however, that it is possible that the effective inducer of the opening of the MTP pore could be the $\mathrm{CoHPO}_{4}$ complex.

\section{Discussion}

As far as the mechanism of action of $\mathrm{Co}^{2+}$ is concerned, the problem is not so easily solved. The mechanism of the opening of the MTP pore has been widely studied. It is very complicated, as it depends on many concomitant factors. An exhaustive explanation of the mechanism has still not been given [12]. For this reason, some proposals regarding the mechanism of action of $\mathrm{Co}^{2+}$ can only be advanced when making analogies with other metals, other proposals can be excluded.

The involvement of $\mathrm{Co}^{2+}$ as a trigger for apoptotic factors, such as the Bcl proteins, normally involved in the opening of the pore during the cell apoptosis would appear to be excluded [13-15].

Recently [16, 17], in this regard, it was demonstrated that, in isolated mitochondria, the effect of Bax proteins (one of the components of the $\mathrm{Bcl}$ family), relative to the opening of the MTP pore, occurs with a protein dose $(200 \mathrm{nM})$ very much higher than that normally present in the (outer) mitochondrial membrane. The authors concluded that, under normal conditions, Bax proteins are not

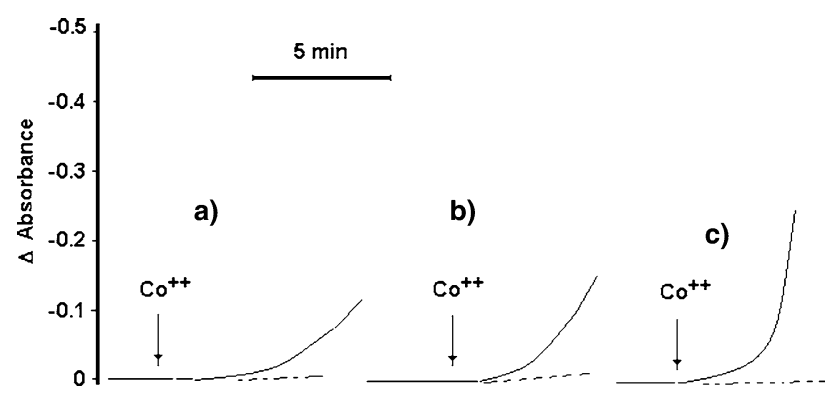

Fig. $4 \mathrm{Co}^{2+}$ induces swelling in mitochondria and this phenomenon is inhibited by cyclosporine. The medium composition was $0.25 \mathrm{M}$ sucrose, $10 \mathrm{mM}$ Hepes/Mops pH 7.4, $1 \mathrm{mM} \mathrm{MgCl} 2,1 \mathrm{mM} \mathrm{Pi}$, and $1 \mathrm{mM}$ succinate. The final concentration of mitochondria was $0.5 \mathrm{mg} /$ $\mathrm{ml}$. Under these conditions, at $540 \mathrm{~nm}$, the addition of $5 \mu \mathrm{M}(a)$, $10 \mu \mathrm{M}(b)$, and $15 \mu \mathrm{M}(c) \mathrm{Co}^{2+}$ induces absorbance quenching, which is inhibited by cyclosporine $(1 \mu \mathrm{g} / \mathrm{ml}$; dotted line $)$, thus indicating that the quenching is due to the opening of a pore involved in the opening of the MTP pore in isolated mitochondria.

Analogously, the experiments of Li et al. [18], regarding the interactions of $\mathrm{Cd}^{2+}$ with mitochondria, exclude interactions between $\mathrm{Cd}^{2+}$ and Bcl- $\mathrm{x}_{\mathrm{L}}$ proteins (proteins of the $\mathrm{Bcl}$ family) and suggest (see below) interaction of $\mathrm{Cd}^{2+}$ with adenine nucleotide translocase (ANT).

All these conclusions are in agreement with those of other research groups that have studied the interactions of metals with isolated mitochondria: in all cases the proposed mechanism for the opening of the MTP pore excludes the involvement of $\mathrm{Bcl}$ proteins. This fact, together with the fact that $\mathrm{Bcl}$ proteins are hydrophobic membrane proteins, leads us to analogous conclusions.

Regarding the opening of the MTP pore, then behavior observed in the experiments is similar for many metals, such as $\mathrm{Zn}^{2+}, \mathrm{Pb}^{2+}, \mathrm{Cd}^{2+}, \mathrm{Hg}^{2+}$, and $\mathrm{Al}^{3+}$, and phenylarsine oxide. The experiments performed on isolated mitochondria and cells show that all metals induce the opening of the MTP pore and that this opening is inhibited by cyclosporine (in the case of $\mathrm{Cd}^{2+}$, however, not all authors are in agreement concerning the cyclosporine sensitivity, as will be discussed below).

With regard to $\mathrm{Zn}^{2+}$ [19] and $\mathrm{Pb}^{2+}$ [20], the opening of the MTP pore has been observed in the presence of micromolar amounts of $\mathrm{Ca}^{2+}$. In our experiments (Fig. 4), $\mathrm{Ca}^{2+}$ was not added, but, by operating under conditions similar to those reported in the literature $[19,20]$ (i.e., $0.25 \mathrm{M}$ sucrose, $10 \mathrm{mM}$ Hepes/Mops $\mathrm{pH} 7.4,5 \mu \mathrm{M}$ $\mathrm{CaCl}_{2}$ ), the addition of small amounts of $\mathrm{Co}^{2+}$ (around $10 \mu \mathrm{M}$ ) induces a swelling, and the swelling is cyclosporine-sensitive (not shown). The above mentioned evidence appears to indicate similar targets for $\mathrm{Co}^{2+}, \mathrm{Zn}^{2+}$, and $\mathrm{Pb}^{2+}$, but in the cases of $\mathrm{Zn}^{2+}$ and $\mathrm{Pb}^{2+}[19,20]$ the authors have not proposed a specific action site for these two metals.

Analogously, in the case of $\mathrm{Cd}^{2+}$, a swelling was observed on isolated mitochondria [18, 21-24] and, also in this case the swelling was facilitated by the presence of $\mathrm{Ca}^{2+}$. However, there is no agreement between the authors concerning the cyclosporine sensitivity. In particular, Pourahmad et al. [21] and Dorta et al. [23], found cyclosporine sensitivity, while Belyaeva et al. [24] and Li et al. [18] do not find cyclosporine sensitivity. As a consequence, different mechanisms for $\mathrm{Cd}^{2+}$ have been proposed; $\mathrm{Li}$ et al. [18] proposed binding of $\mathrm{Co}^{2+}$ to the thiol groups in ANT.

Both the swelling and the cyclosporine sensitivity were verified in the case of $\mathrm{Al}^{3+}$ and $\mathrm{Hg}^{2+}[11,21]$. In the case of $\mathrm{Al}^{3+}$ "binding of aluminum to the inner mitochondrial membrane, most likely at the level of adenine translocase (ANT)" has been proposed [11].

In the case of phenylarsine oxide, the cyclosporine sensitivity has been verified [25], and it has been proposed 
that phenylarsine (and diamide) cause an intramolecular cross-linking between the thiol groups, i.e., Cys ${ }^{160}$ and $\mathrm{Cys}^{257}$ [25] in ANT. This cross-linking stabilizes the " $c$ ", conformation of ANT. This stabilization should enhance the sensitivity of the MTP pore to the endogenous $\mathrm{Ca}^{2+}$ [25].

The strong analogy between $\mathrm{Co}^{2+}, \mathrm{Al}^{3+}, \mathrm{Cd}^{2+}$, and phenylarsine oxide could suggest similar molecular behavior, i.e., interaction of $\mathrm{Co}^{2+}$ with the thiol groups in ANT.

\section{References}

1. Hervouet E, Pecina P, Demont J, Vojtiskova A, Simonnet H, Houstek J, Godinot C (2006) Biochem Biophys Res Commun 344:1086-1093

2. Fleury C, Petit A, Mwale F, Antoniu J, Zukor DJ, Tabrizian M, Huk OL (2006) Biomaterials 27:3351-3360

3. Coddou C, Lorca RA, Acuna-Castillo C, Grauso M, Rassendren F, Huidobro-Toro JP (2005) Toxicol Appl Pharmacol 202:121131

4. Catelas I, Petit A, Vali H, Fragiskatos C, Meilleur R, Zukor DJ, Antoniu J, Huk OL (2005) Biomaterials 26:2441-2453

5. Huk OL, Catelas I, Mwale F, Antoniou J, Zukor DJ, Petit A (2004) J Arthroplasty 19:84-87

6. Robertson JD, Orrenius S (2000) Crit Rev Toxicol 30:609-627

7. Azzone GF, Pozzan T, Bragadin M, Miconi V (1979) J Biol Chem 254:10213-10219

8. Lowry OH, Rosebrough NJ, Farr AL, Randall RJ (1951) J Biol Chem 193:265-275
9. Mitchell P (1979) Science 206:1148-1159

10. Smith RM, Martell AE (1976) In: Smith RM, Martell AE (eds) Critical stability constants, vol 4. Plenum, New York, p 54

11. Toninello A, Clari G, Mancon M, Tognon G, Zatta P (2000) J Biol Inorg Chem 5:612-623

12. Zoratti M, Szabò I, De Marchi U (2005) Biochim Biophys Acta $1706: 40-52$

13. Kaufmann T, Schinzel A, Borner C (2004) Trends Cell Biol $14: 8-12$

14. Desagher S Martinou JC (2000) Trends Cell Biol 10:369-377

15. Sharpe JC, Arnoult D, Youle RJ (2004) Biochim Biophys Acta 1644:107-113

16. Campello S, De Marchi U, Szabo I, Tombola F, Martinou JC, Zoratti M (2005) FEBS Lett 579:3695-3700

17. De Marchi U, Campello S, Szabo I, Tombola F, Martinou JC Zoratti M (2004) J Biol Chem 279: 37415-37422

18. Li M, Xia T, Jiang CS, Li LJ, Fu JL, Zhou ZC (2003) Toxicology 194:19-33

19. Jang D, Sullivan PG, Sensi SL, Steward O, Weiss JH (2001) J Biol Chem 276:47524-47529

20. He L, Poblenz AT, Medrano CJ, Fox DA (2000) J Biol Chem 275:12175-12184

21. Pourahmad J, Mihailovic A, O'Brien PJ (2001) Adv Exp Med Biol 500:249-252

22. Belyaeva EA, Glazunov VV, Nikitina ER, Korotkov SM (2001) J Bioenerg Biomembr 33:303-318

23. Dorta DJ, Leite S, De Marco KC, Prado IM, Rodriguez T, Mingatto FE, Uyemura SA, Santos AC, Curti C (2003) J Inorg Biochem 97:251-257

24. Belyaeva EA, Glazunov VV, Korotkov SM (2004) Acta Biochim Pol 51:19-33

25. McStay GP, Clarke S, Halestrap A (2002) Biochem J 367:541548 